Proceedings

\title{
Urea-Functionalized Chitosan as Efficient and Recoverable Organocatalyst for the Convenient Synthesis of Pyrimido[4,5-b]quinoline-2,4-dione Derivatives $^{\dagger}$
}

\author{
Negin Rostami, Mohammad G. Dekamin * and Ehsan Valiey \\ Pharmaceutical and Heterocyclic Compounds Research Laboratory, Department of Chemistry, Iran \\ University of Science and Technology, Tehran 16846-13114, Iran; \\ * Correspondence: mdekamin@iust.ac.ir; Tel.: +98-21-77-240-284; Fax: +98-21-73-021-584 \\ + Presented at the 24th International Electronic Conference on Synthetic Organic Chemistry, 15 November- \\ 15 December 2020; Available online: https://ecsoc-24.sciforum.net/.
}

Published: date

\begin{abstract}
Urea-functionalized chitosan (Urea-Pr-Cs) was used as a new and efficient catalyst for the synthesis of pyrimido[4,5- $b$ ]quinoline-2,4-dione derivatives in aqueous media. In this regard, some of the heterocyclic pyrimidine derivatives have been synthesized by using reflux conditions in a four-component, efficient and efficient manner, with the reaction of baritrionic acid condensation, aldehyde derivatives, specific amines, and dimedone in $\mathrm{EtOH} / \mathrm{H}_{2} \mathrm{O}$ as solvent. This method has the prominent features such as green solvent, high to quantitative yields of the favorable products, easy product separation, no purification with chromatography column, heterogeneous catalyst and easy recyclability of catalyst. In this methodology, the ideal conditions for the reaction are shown.
\end{abstract}

Keywords:

\section{Introduction}

Multicomponent reactions (MCRs) are atom-efficient routes to achieve complex chemicals which simplifies procedures because they avoid the isolation of intermediates [1]. Indeed, this methodology, allowing multiple bond formation in a single synthetic step [2,3].

Urea-functionalized chitosan (Urea-Pr-Cs), containing Brønsted base centers, was designed as a highly efficient, selective and recoverable organocatalyst for the four-component synthesis of biomedical derivatives of pyrimido[4,5-b]quinoline-2,4-dione. These derivatives are vital class of $N$ heterocyclic and biologically-active compounds [4].

In continuation of previous our works in the preparation of new heterogeneous catalyst for organic transformations [5-12], Herein, we report our results for a one-pot synthesis of pyrimido[4,5b]quinoline-2,4-dione derivatives using Urea-Pr-Cs (1) as a highly efficient metal free organocatalyst. The reported protocol illustrates several prominent features such as high yield, purification of products without using chromatography columns and the use of heterogeneous catalyst in available and inexpensive which is also environmentally friendly solvent. 


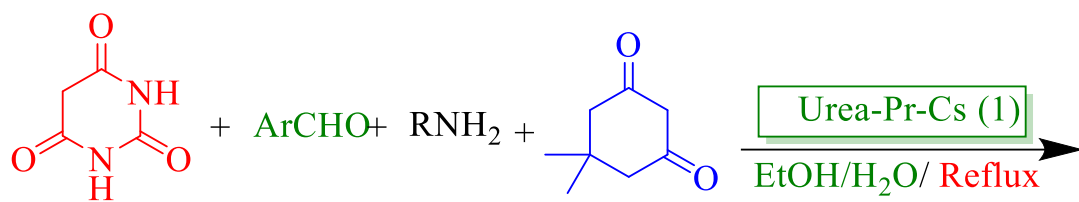

(2)<smiles>CC1(C)CC(=O)C2=C(C1)Nc1[nH]c(=O)[nH]c(=O)c1C2[Al]</smiles>

(6)

Scheme 1. Synthesis of pyrimidine derivatives catalyzed by Cs-Pr-Urea (1).

\section{Experimental Section}

\subsection{General}

\section{Reagents and Apparatus}

All chemical reagents were purchased from Merck. To determine the completion of the reaction, analytical thin-layer chromatography (TLC) was performed on pre-coated silica-gel plates (Merck Silica Gel F254). Product stains were detected either under UV light or by placing in an iodine chamber. Also, melting points were determined in open capillaries using an Electrothermal 9100 apparatus.

General Procedure for the Synthesis of Pyrimido[4,5-b]quinoline-2,4-dione (6a-d)

In a round-bottomed flask, baritrionic acid $(\mathbf{2}, 1 \mathrm{mmol})$, various aryl aldehydes (3a-d, $1 \mathrm{mmol})$, various amine sources $(4,1 \mathrm{mmol})$, dimedon $(5,1 \mathrm{mmol})$ and $10 \mathrm{mg}$ of Urea-Pr-Cs (1) were added to $\mathrm{EtOH} / \mathrm{H}_{2} \mathrm{O}(2 \mathrm{~mL})$. The obtained mixture was stirred under reflux conditions. After completion of the reaction monitored by TLC (EtOAc/n-hexane 1/3), EtOH $(3 \mathrm{~mL})$ was added to the crude reaction mixture and heated to separate the catalyst $\mathbf{1}$ by filteration. The filtrate was kept at ambient temperature to give pure products. Also, the separated catalyst was dried and used in the next times.

\section{Results and Discussion}

The catalytic activity of Urea-Pr-Cs (1) was evaluated in the green synthesis of pyrimido[4,5$b$ ]quinoline-2,4-dione derivatives by condensing baritrionic acid, aryl aldehyde derivatives, amine and dimedone in water under reflux conditions. According to Scheme 2, using a small amount of Urea-Pr-Cs (1) as heterogeneous catalyst (about $10 \mathrm{mg}$ ), the desired products were synthesized with high yield and in short times. The ability to easily separate and recycle the catalyst from the reaction mixture with minimal effort is another advantage of this bio-based organocatalyst. This catalyst reused for at least five runs without significant loss of its catalytic activity. 
<smiles>CC1(C)CC(=O)C2=C(C1)Nc1[nH]c(=O)[nH]c(=O)c1C2c1ccc(Cl)cc1</smiles>

6a yield:95\%<smiles>CC1(C)CC(=O)C2=C(C1)Nc1[nH]c(=O)[nH]c(=O)c1C2c1ccccc1</smiles>

6c yield:94\%<smiles>COc1ccc(C2C3=C(CC(C)(C)CC3=O)Nc3[nH]c(=O)[nH]c(=O)c32)cc1</smiles>

6b yield:92\%<smiles>CC1(C)CC(=O)C2=C(C1)Nc1[nH]c(=O)[nH]c(=O)c1C2c1ccc([N+](=O)[O-])cc1</smiles>

6d yield: $90 \%$

Scheme 2. Scope of pyrimido[4,5- $b$ ]quinoline-2,4-dione derivatives (6a-d) synthesis catalyzed by Urea-Pr-Cs (1).

\section{Conclusion}

In summary, the new bio-based urea-functionalized chitosan (Urea-Pr-Cs) catalyst was used for four component reaction of baritrionic acid, aryl aldehyde derivatives, amine and dimedone in $\mathrm{EtOH} / \mathrm{H}_{2} \mathrm{O}$ under reflux conditions to afford biologically-ctive pyrimido[4,5-b]quinoline-2,4-dione compounds. The advantages of this method are low loading of transition metal-free catalyst, easy separation and reusability of catalyst, high-to-excellent yields of products, use of green solvent, simple and chromatography free work-up procedure and mild reaction conditions. Furthermore, this organocatalyst was recovered and reused at least five times without a considerable decrease in its activity.

Acknowledgments: We are grateful for the financial support from The Research Council of Iran University of Science and Technology (IUST), Tehran, Iran (Grant No. 160/19108).

\section{References}

1. Goddard, J.-P.; Malacria, M.; Ollivier, C. Multi-Component Reactions in Molecular Diversity; Wiley Online Library: Hoboken, NJ, USA, 2020.

2. Müller, T.J. Multicomponent reactions in the synthesis of heterocycles. Chem. Heterocycl. Compd. 2017, 53, 381.

3. Dekamin, M.G.; Eslami, M.; Maleki, A. Potassium phthalimide-N-oxyl: A novel, efficient, and simple organocatalyst for the one-pot three-component synthesis of various 2-amino-4H-chromene derivatives in water. Tetrahedron 2013, 69, 1074-1085. 
4. Dhameliya, T.M.; Donga, H.A.; Vaghela, P.V.; Panchal, B.G.; Sureja, D.K.; Bodiwala, K.B.; Chhabria, M.T. A decennary update on applications of metal nanoparticles (MNPs) in the synthesis of nitrogen-and oxygen-containing heterocyclic scaffolds. RSC Adv. 2020, 10, 32740-32820.

5. Akbari, A.; Dekamin, M.G.; Yaghoubi, A.; Naimi-Jamal, M.R. Novel magnetic propylsulfonic acidanchored isocyanurate-based periodic mesoporous organosilica (Iron oxide@ PMO-ICS-PrSO 3 H) as a highly efficient and reusable nanoreactor for the sustainable synthesis of imidazopyrimidine derivatives. Sci. Rep. 2020, 10, 1-16.

6. Alirezvani, Z.; Dekamin, M.G.; Valiey, E. New Hydrogen-Bond-Enriched 1, 3, 5-Tris (2-hydroxyethyl) Isocyanurate Covalently Functionalized MCM-41: An Efficient and Recoverable Hybrid Catalyst for Convenient Synthesis of Acridinedione Derivatives. ACS Omega 2019, 4, 20618-20633.

7. Valiey, E.; Dekamin, M.G.; Alirezvani, Z. Melamine-modified chitosan materials: An efficient and recyclable bifunctional organocatalyst for green synthesis of densely functionalized bioactive dihydropyrano [2, 3-c] pyrazole and benzylpyrazolyl coumarin derivatives. Int. J. Biol. Macromol. 2019, 129, 407-421.

8. Dekamin, M.; Kazemi, E.; Karimi, Z.; Mohammadalipoor, M.; Naimi-Jamal, M.R. Chitosan: An efficient biomacromolecule support for synergic catalyzing of Hantzsch esters by CuSO4. Int. J. Biol. Macromol. 2016, 93, 767-774.

9. Alirezvani, Z.; Dekamin, M.G.; Valiey, E. $\mathrm{Cu}(\mathrm{II})$ and magnetite nanoparticles decorated melaminefunctionalized chitosan: A synergistic multifunctional catalyst for sustainable cascade oxidation of benzyl alcohols/Knoevenagel condensation. Sci. Rep. 2019, 9, 17758.

10. Dekamin, M.G.; Kazemi, E.; Karimi, Z.; Mohammadalipoor, M.; Naimi-Jamal, M.R. Chitosan: An efficient biomacromolecule support for synergic catalyzing of Hantzsch esters by CuSO4. Int. J. Biol. Macromol. 2016, 93, 767-774.

11. Dekamin, M.G.; Azimoshan, M.; Ramezani, L. Chitosan: A highly efficient renewable and recoverable biopolymer catalyst for the expeditious synthesis of $\alpha$-amino nitriles and imines under mild conditions. Green Chem. 2013, 15, 811-820.

12. Akbari, A.; Dekamin, M.G.; Yaghoubi, A.; Naimi-Jamal, M.R. Novel magnetic propylsulfonic acidanchored isocyanurate-based periodic mesoporous organosilica (Iron oxide@PMO-ICS-PrSO3H) as a highly efficient and reusable nanoreactor for the sustainable synthesis of imidazopyrimidine derivatives. Sci. Rep. 2020, 10, 10646.

Publisher's Note: MDPI stays neutral with regard to jurisdictional claims in published maps and institutional affiliations.

(C) 2020 by the authors. Submitted for possible open access publication under the terms and conditions of the Creative Commons Attribution (CC BY) license (http://creativecommons.org/licenses/by/4.0/). 